\title{
QUALIDADE DA CARNE OVINA SUBMETIDA AO PROCESSO DE SALGA ${ }^{1}$
}

\author{
Américo Garcia da SILVA SOBRINHO², Nivea Maria Brancacci Lopes ZEOLA ${ }^{3, *}$,
}

Hirasilva Borba Alves de SOUZA , Tânia Mara Azevedo de LIMA ${ }^{5}$

\section{RESUMO}

Foi estudada a qualidade da carne ovina submetida a diferentes teores de sal no processo de salga, utilizando-se 18 pernas desossadas provenientes de cordeiros $1 / 2$ Ile de France $1 / 2$ Ideal, machos inteiros, com peso vivo médio de 30kg. Os tratamentos constituíram-se em: $\mathrm{T}_{1}$ - controle; $\mathrm{T}_{2}$ salga a $15 \%$ e $\mathrm{T}_{3}$ - salga a $20 \%$. As mantas de carne foram lavadas rapidamente em água corrente para remoção do excesso de sal e secadas à sombra. As análises foram realizadas antes da salga e 67 horas após o início da mesma. As perdas de peso ao cozimento, maciez, cor, umidade $(2,4,16,28,40$ e 67 horas após o início da salga) e número de TBARS, substâncias reativas ao ácido 2 -tiobarbitúrico, foram realizadas no músculo Semimembranosus. Os diferentes teores de sal não influenciaram $(P>0,05)$ na luminosidade da carne, entretanto os teores de vermelho e amarelo, que foram influenciados $(\mathrm{P}<0,05)$. As perdas de peso ao cozimento e a umidade foram menores $(\mathrm{P}<0,05)$ nas carnes salgadas. A carne dos tratamentos controle e com $15 \%$ de salga, apresentou maior $(\mathrm{P}<0,05)$ maciez, em relação à carne do tratamento com $20 \%$ de salga. A carne que foi submetida a $20 \%$ de salga, apresentou maior oxidação lípidica, não diferindo da salga a $15 \%$. A salga a $15 \%$ forneceu melhores resultados com valores de perdas de peso ao cozimento, cor, umidade e número de TBARS semelhante a salga a $20 \%$, porém com maior maciez.

Palavras-chave: carne; ovinos; salga.

\section{SUMMARY}

MEAT QUALITY OF LAMB SUBMITTED TO SALTING. The effects was studied of different salt quantities during salting process in qualitative parameters of sheep meat. Were used 18 boned legs from ram lambs $1 / 2$ Ile de France $1 / 2$ Ideal, slaughtered with $30 \mathrm{~kg}$ of body weight. The treatments were: $\mathrm{T}_{1}$ - control; $\mathrm{T}_{2}$ - salting at $15 \% ; \mathrm{T}_{3}$ - salting at $20 \%$. Meat slices were washed with water to remove salt excess and dried in shadow. Analysis were done before salting and 67 hours after salting. Cooking losses, tenderness, colour, humidity $(2,4,16,28,40$ and 67 hours after the beginning of the process) and number of TBARS (2-tiobarbituric acid reactive substances) were done on Semimembranosus muscle. Different quantities of salt had no effect on meat lightness $(P>0.05)$, although redness and yellowness were affected $(\mathrm{P}<0.05)$. Cooking losses and humidity were lower $(\mathrm{P}<0.05)$ for salted meat. The tenderness was higher $(\mathrm{P}<0.05)$ for meats submitted to control treatment and $15 \%$ of salting, compared to meats salted with $20 \%$. Meats submitted to $20 \%$ of salting showed higher number of TBARS, not differing $15 \%$ of salting. The utilization of $15 \%$ of salting showed better results, with cooking losses, colour, humidity and number of TBARS similar to salting at $20 \%$, however the meat was more tender. Keywords: meat; ovine; salting process.

\section{1 - INTRODUÇÃO}

A salga é um método empregado na conservação de carnes e derivados, com certa tradição em algumas regiões brasileiras, sendo de grande importância onde a refrigeração torna-se difícil, como em muitas cidades do Nordeste brasileiro [9].

Uma importante função do sal na indústria de produtos cárneos, é a extração das proteínas miofibrilares. A extração e a solubilização dessas proteínas musculares contribuem para a emulsificação das gorduras e para aumentar sua capacidade de retenção de água, reduzindo as perdas de peso ao cozimento, contribuindo para melhorar a qualidade e a textura do produto [6, 21].

\footnotetext{
1. Recebido para publicação em 09/09/2002. Aceito para publicação em 06/07/2004 (000971).

2. Departamento de Zootecnia-FCAV/UNESP, Jaboticabal, SP, Brasil. Email: americo@fcav.unesp.br

3. Curso de Pós-Graduação em Produção Animal-FCAV/UNESP, Jaboticabal, SP, Brasil.

4. Departamento de Tecnologia - FCAV/UNESP, Jaboticabal, SP, Brasil

5. Laboratório de Tecnologia dos Produtos de Origem Animal - FCAV/ UNESP, Jaboticabal, SP, Brasil

* A quem a correspondência deve ser enviada.
}

A ação do sal também está relacionada com a inibição da multiplicação de microrganismos [3, 11]. O crescimento de algumas bactérias é inibido à baixas concentrações de sal, como $2 \%$, embora outras bactérias, leveduras e fungos sejam capazes de crescer em concentrações salinas muito elevadas, incluindo o ponto de saturação. Alguns microrganismos (halófilos) crescem apenas em meios com altas concentrações de sal, morrendo rapidamente quando colocados em meios com menos de 10\% de cloreto de sódio [9].

Em associação com o calor, o sal tem a propriedade de desidratar a carne, provocando diminuição da umidade e da atividade de água. Entretanto, nestas condições, o produto cárneo pode sofrer deterioração oxidativa, promovendo rancidez dos lipídios [10, 21], ou seja, o sal torna-se um pró-oxidante da gordura provocando a ativação da lipoxidase do músculo [17].

A rancidez oxidativa é proveniente da oxidação dos ácidos graxos polinsaturados presentes na carne, sendo que a concentração de malonaldeído, produto secundário desta oxidação, pode ser determinada através do teste do ácido tiobarbitúrico (TBARS). O teor de rancidez é normalmente expresso em número de TBARS, ou seja, em mg malonaldeído/kg de amostra de carne (Gray \& Pearson citados por TRINDADE) [26]. Neste contexto, a carne ovina sendo rica em ácidos graxos saturados, como o mirístico, o palmítico e o esteárico, 
quando comparada com carnes de aves e/ou suínos, que apresentam maior quantidade de ácidos graxos insaturados, apresenta-se menos susceptivel à este tipo de rancidez [15].

A importância da oxidação lipídica em carnes tem sido enfatizada recentemente, pois o consumo de alimentos rancificados, pode induzir a doenças coronarianas, câncer e derrame cerebral (ADDIS citado por LIRA et al. [13]).

Neste contexto, propriedades da carne, como cor, maciez e perdas de peso ao cozimento, determinam sua utilidade para comercialização, aparência e adaptabilidade aos processamentos industriais [4].

A cor da carne é o fator de qualidade mais importante que o consumidor pode apreciar no momento da compra, constituindo o critério básico para sua seleção, a não ser que outros fatores, como o odor, sejam marcadamente deficientes.

A utilização de sais produz alteração na mioglobina do músculo, pois o ferro é oxidado, originando a metamioglobina, de cor marron, associada pelos consumidores a carnes estocadas por longos períodos [20].

Considerando tais peculiaridades este trabalho objetivou estudar o efeito da salga nos parâmetros qualitativos da carne ovina.

\section{2 - MATERIAL E MÉTODOS}

\section{1 - Local}

Este trabalho foi desenvolvido na Faculdade de Ciências Agrárias e Veterinárias - FCAV/UNESP, Jaboticabal, SP. A fase de campo e o abate dos animais foram desenvolvidos nas dependências do Setor de Ovinocultura e as análises físico-químicas no Laboratório de Tecnologia dos Produtos de Origem Animal, pertencentes aos Departamentos de Zootecnia e Tecnologia, respectivamente.

\section{2 - Salga}

O método utilizado foi a salga a seco, com inclusão de sal de granulação média, na base de 15 e 20\% do peso da peça.

Foram utilizadas 18 pernas provenientes de cordeiros $1 / 2$ Ile de France $1 / 2$ Ideal, machos inteiros, abatidos aos $30 \mathrm{~kg}$ de peso vivo. As pernas foram desossadas e a carne salgada, mantida em pilhas e tombada quatro vezes em intervalos de 30 minutos e deixada em repouso por 4 horas. As peças foram lavadas rapidamente em água corrente para remoção do excesso de sal e secadas à sombra. As análises foram realizadas no músculo Semimembranosus antes da salga e 67 horas após o início da salga. A umidade foi avaliada em intervalos de 2 , $4,16,28,40$ e 67 horas após o início da salga.

\section{3 - Delineamento experimental}

O delineamento experimental foi o inteiramente casualizado, com três tratamentos e seis repetições. Os fatores avaliados foram carne sem salga, salga seca a $15 \%$ e salga seca a $20 \%$, constituindo os tratamentos $\mathrm{T}_{1}$ - controle; $\mathrm{T}_{2}$ - salga a $15 \%$ e $\mathrm{T}_{3}$ - salga a $20 \%$, respectivamente.

As comparações dos contrastes entre médias dos tratamentos foram feitas pelo teste de Tukey a 5\% e as análises de variância segundo procedimentos do ESTAT [5].

\section{4 - Procedimento para análises físico-químicas}

Para determinação das perdas de peso ao cozimento, maciez, cor, umidade e número de substâncias reativas ao ácido 2 -tiobarbitúrico foram retiradas amostras do músculo Semimembranosus. As perdas de peso ao cozimento foram determinadas conforme metodologia descrita por CORTE, FELÍCIO \& CIA [2]. As amostras foram pesadas em bandejas com grelha e levadas a $170^{\circ} \mathrm{C}$, até que a temperatura interna atingisse $75^{\circ} \mathrm{C}$. Posteriormente, foram retiradas do forno e pesadas novamente, obtendo-se por diferença as perdas de peso ao cozimento. Das amostras assadas foram retiradas sub-amostras de $2 \mathrm{~cm}$ de diâmetro para a determinação da maciez em Texture Analyser acoplado ao dispositivo Warner - Bratzler, obtendo-se a força de cisalhamento da amostra em kg [2].

Para determinação da cor utilizou-se o colorímetro Minolta CR-200, o qual determina a cor da carne através das coordenadas $\mathrm{L}^{*}, \mathrm{a}^{*} \mathrm{e} \mathrm{b}^{*}$, relacionadas à luminosidade, teor de vermelho e teor de amarelo, respectivamente [14].

Na determinação da umidade as amostras foram pesadas antes e após a liofilização para posterior determinação da $1{ }^{\mathrm{a}} \mathrm{MS}$ (matéria seca), que corresponde à perda de umidade da amostra realizada em estufa à temperatura de $65^{\circ} \mathrm{C}$. Posteriormente, determinou-se a $2^{\mathrm{a}} \mathrm{MS}$, que corresponde à perda de umidade da amostra realizada em estufa a temperatura de $105^{\circ} \mathrm{C}$, e por diferença calculou-se a umidade, conforme metodologias descritas por SILVA [22].

Para o teor de rancidez dos lipídios, foi realizado o teste do ácido 2-tiobarbitúrico (TBARS), pesando-se $5 \mathrm{~g}$ de amostra homogeneizada e adicionando-se $25 \mathrm{~mL}$ de TCA (tetrametoxipropano) a 7,5\%. Posteriormente procedeu-se homogeneização por 1 minuto com filtragem em tubo corning. Acrescentou-se em tubo de ensaio $4 \mathrm{~mL}$ do filtrado, $1 \mathrm{~mL}$ de TCA (ácido tricloroacético) e $5 \mathrm{~mL}$ de TBA (ácido tiobarbitúrico). Os tubos foram colocados em água fervente por 40 minutos. Após esfriarem foi realizada a leitura em espectofotômetro a 538nm, acompanhada de curva padrão, de acordo com o método descrito por PIKUL, LESZCZYNSKI \& KUMMEROW [18].

\section{3 - RESULTADOS E DISCUSSÃO}

A Tabela 1 apresenta medidas de cor da carne (L*, $a^{*}$ e b*) submetidas a diferentes teores de sal no processo de salga.

Os diferentes teores de sal não influenciaram $(\mathrm{P}>0,05)$ na luminosidade da carne, com valor médio de 38,69. Entretanto, carnes que não receberam salga apresentaram maiores teores de vermelho e amarelo $(\mathrm{P}<0,05)$, 
em relação às salgadas. De acordo com SABADINI et al. [20], a salga tende a diminuir a cor vermelha da carne, devido às modificações que ocorrem na mioglobina. Em contrapartida, FAROUK \& SWAN [8] ao estudarem o efeito da salga no pré-rigor em bovinos, observaram que as carnes que foram submetidas a salga, apresentaram-se mais vermelhas, com valores de a* entre 15 e 16 .

TABELA 1. Cor da carne de cordeiros (L*, a* e b*) submetida a diferentes teores de sal

\begin{tabular}{|c|c|c|c|c|}
\hline \multirow[t]{2}{*}{ Cor } & \multicolumn{3}{|c|}{ Teor de sal (\%) } & \multirow{2}{*}{$\begin{array}{l}\text { CV } \\
(\%)\end{array}$} \\
\hline & 0 & 15 & 20 & \\
\hline $\mathrm{L}^{*}$ & $38,29^{a}$ & $39,71^{a}$ & $38,07^{a}$ & 4,02 \\
\hline$a^{*}$ & $14,79^{\mathrm{a}}$ & $7,34^{b}$ & $7,68^{\mathrm{b}}$ & 9,25 \\
\hline$b^{*}$ & $3,40^{\mathrm{a}}$ & $0,59^{b}$ & $0,38^{b}$ & 43,13 \\
\hline
\end{tabular}

Em ovinos são descritos valores 31,36 a 38,0 para $L^{*}$, de 12,27 a 18,01 para a* e de 3,34 a 5,65 para b* (Souza citado por FARIA et al. [7]). Neste experimento, alguns valores enquadram-se nestes intervalos, porém os valores de $\mathrm{b}^{*}$ foram muito inferiores nos tratamentos $\mathrm{T}_{1}$ e $\mathrm{T}_{2}$.

SILVA SOBRINHO [24], ao avaliar a cor da carne em ovinos neozelandeses de diferentes genótipos, obteve valores $\mathrm{L}^{*}$, a* e b* de 37,50; 7,83 e 4,30, respectivamente. Estudando características físico-químicas da carne de cordeiros $1 / 2$ Santa Inês $1 / 2$ Bergamácia abatidos aos $25 \mathrm{~kg}$, SOUZA et al. [25] encontraram os valores de 32,65 para L* e 15,34 para a*. Em trabalho recente, ZEOLA [27] avaliando a cor da carne de cordeiros Morada Nova submetidos a dietas com diferentes níveis de concentrado, registrou valores $L^{*}, a^{*}$ e b* de 40,$46 ; 14,62$ e 1,11 , respectivamente. Todos os autores citados avaliaram a cor no músculo Semimembranosus.

A Tabela 2 apresenta dados de perdas de peso ao cozimento, força de cisalhamento, umidade e número de TBARS da carne de cordeiros submetida a diferentes teores de sal no processo de salga.

TABELA 2. Perdas de peso ao cozimento, força de cisalhamento, umidade e número $\left(\mathrm{N}^{\circ}\right)$ de TBARS da carne de cordeiros submetida a diferentes teores de sal

\begin{tabular}{lcccc}
\hline \multirow{2}{*}{ Parâmetro } & \multicolumn{3}{c}{ Teor de sal (\%) } & CV \\
\cline { 2 - 4 } & 0 & 15 & 20 & $(\%)$ \\
\hline Perdas de peso ao cozimento (\%) & $37,27^{\mathrm{a}}$ & $16,30^{\circ}$ & $18,86^{\circ}$ & 9,09 \\
Força de cisalhamento (kg) & $8,21^{\mathrm{b}}$ & $11,66^{\mathrm{b}}$ & $16,04^{\mathrm{a}}$ & 18,24 \\
Umidade (\%) & $72,22^{\mathrm{a}}$ & $40,35^{\mathrm{b}}$ & $40,51^{\mathrm{b}}$ & 1,27 \\
$\mathrm{~N}^{\circ}$ de TBARS (mg malonaldeído/kg) & $2,26^{\mathrm{b}}$ & $3,07^{\mathrm{ab}}$ & $3,58^{\mathrm{a}}$ & 18,58 \\
\hline a,b Letras distintas na mesma linha diferem significativamente (P<0,05) entre si pelo \\
teste de Tukey \\
CV - coeficiente de variação
\end{tabular}

Os diferentes teores de sal influenciaram $(\mathrm{P}<0,05)$ os parâmetros qualitativos da carne ovina. As perdas de peso ao cozimento foram menores $(\mathrm{P}<0,05)$ nas carnes salgadas, com média de $17,58 \%$. De acordo com SILVA [23] o sal extrai e solubiliza proteínas miofibri- lares da carne, e estes processos contribuem para a emulsificação das gorduras e para aumentar a capacidade de retenção de água, reduzindo as perdas ao cozimento.

A força de cisalhamento apresentou melhores resultados nas carnes dos tratamentos controle $(8,21 \mathrm{~kg})$ e com $15 \%$ de salga $(11,66 \mathrm{~kg})$. O tratamento com $20 \%$ de salga apresentou menor maciez $(16,04 \mathrm{~kg})$ da carne diferindo $(\mathrm{P}<0,05)$ dos tratamentos $\mathrm{T}_{1}$ e $\mathrm{T}_{2}$. Em todos os tratamentos a carne não apresentou boa maciez, considerando o fato de os valores de força de cisalhamento obtidos serem superiores ao valor de referência de $8 \mathrm{~kg}$ (SWAN et al., citado por NOGUEIRA et al. [16]).

A umidade foi menor $(\mathrm{P}<0,05)$ nas carnes que receberam salga, com média de $40,43 \%$, em relação as que não receberam, cujo valor foi de $72,22 \%$.

FURTADO et al. [9] ao avaliarem a qualidade da carne caprina salgada a $15 \%$, observaram valores de umidade de 72 e 54,3\%, no momento da salga e 72 horas após o início da salga, respectivamente. Em outro experimento, FURTADO et al. [10] avaliaram o efeito da castração e da salga a $15 \%$ na qualidade da carne caprina, obtendo valores de umidade para animais inteiros de 71,9 e $56,9 \%$, nos tratamentos sem adição de salga e com adição de salga, respectivamente. Para animais castrados, os valores foram $73,1 \%$ (sem salga) e $54,2 \%$ (com salga). De acordo com os autores o teor de umidade diminuiu 15 e 18,9\% na carne dos animais inteiros e castrados, respectivamente.

A incorporação do sal provocou mudanças bioquímicas na carne em relação à rancificação das gorduras. O tratamento com salga a $20 \%$ apresentou maior número de TBARS $(3,58 \mathrm{mg} / \mathrm{kg})$ não diferindo $(\mathrm{P}>0,05)$ do tratamento com salga a $15 \%(3,07 \mathrm{mg} / \mathrm{kg})$. Entretanto, menor número de TBARS foi observado na carne do tratamento controle $(2,26 \mathrm{mg} / \mathrm{kg})$, o qual não diferiu $(\mathrm{P}>0,05)$ do tratamento com salga a $15 \%$. Corroborando com tais resultados, ARNAU, GOU \& COMAPOSADA [1] descreveram que quanto maiores as concentrações de sal em produtos curados, maior é a oxidação destes produtos.

Os valores obtidos neste trabalho foram superiores aos do intervalo citado por GREENE \& CUMUZE [12], decorrente da percepção de rancidez na carne cozida em análise sensorial, que variou de 0,6 a 2,0.

Estudando a qualidade da carne caprina salgada, FURTADO et al. [9] encontraram número de TBARS (mg/ $\mathrm{kg}$ ) de 0,84 e 1,06, antes da salga e 72 horas após o início da salga, respectivamente, retratando o incremento na oxidação dos lipídios da carne. Em trabalho mais recente, LIRA et al. [13] avaliaram a oxidação lipídica em carne bovina processada (carne-de-sol), observando diferenças $(\mathrm{P}<0,05)$ no número de TBARS $(\mathrm{mg} / \mathrm{kg})$ entre as carnes antes do processamento e após a salga, com valores de 0,074 e 0,101, respectivamente.

\section{4 - CONCLUSÕES}

Carnes de ovinos submetidas a salga a $15 \%$ apresentaram maior maciez do que àquelas que foram sub- 
metidas a salga a $20 \%$, razão pela qual a salga a $15 \%$ seria recomendada, embora os valores de perdas de peso ao cozimento, cor, umidade e número de TBARS tenham sido semelhantes.

\section{5 - REFERÊNCIAS BIBLIOGRÁFICAS}

[1] ARNAU, J.; GOU, P.; COMAPOSADA, J. Dry-cured ham and fermented meat products: formulating for flavour. Brazilian Journal of Food Technology, v.6, p.126136, 2003.

[2] CORTE, O.O.; FELÍCIO, P.E.; CIA, G. Sistematização da avaliação final de bovinos e bubalinos. III. Qualidade da carne. Boletim Técnico do CTC, Campinas, n. 3, p. 66-76, 1979.

[3] COUTRON-GAMBOTTI,C.; GANDEMER, G.; ROUSSET, S.; MAESTRINI, O.; CASABIANCA, F. Reducing salt content of dry-cured ham: effect on lipid composition and sensory attributes. Food Chemistry, n. 64, p. 13$19,1999$.

[4] DABÉS, A.C. Propriedades da carne fresca. Revista Nacional da Carne, v.25, n.288, p.32-40, 2001.

[5] ESTAT - Sistema para análises estatísticas, versão 2.0, Unesp - Jaboticabal, 1992.

[6] GAVA, A.J. Princípios de tecnologia de alimentos. São Paulo: Nobel, 1941. 284p.

[7] FARIA, P.B.; BRESSAN, M.C.; ODA, S.H.I.; MIGUEL, G.Z.; REBELLO, F.F.P.; VIEIRA, J.O.; PEREZ, J.R.O. Características de $\mathrm{pH}$ e cor (CIELAB) de carne de 0capivaras (Hydrochaeris hydrochaeris L. 1766) nas primeiras $24 \mathrm{~h}$ post mortem. In: SIMPÓSIO LATINO AMERICANO DE CIÊNCIA DE ALIMENTOS, 4., 2001, Campinas. Anais...Campinas, 2001. p.157.

[8] FAROUK, M.M.; SWAN, J.E. Effect of pH at time of salting on the functional properties of pre-rigor beef. Meat Science, v. 45, n. 4, p. 463-472, 1997.

[9] FURTADO, S.M.B.; SHIMOKOMAKI, S.; ROMANELLI, P.F.; RODRIGUES-AWAYA, D.B. Avaliação da qualidade da carne caprina salgada. Higiene Alimentar, v. 5, n. 18, p. 34-38, 1991.

[10] FURTADO, S.M.B.; ROMANELLI, P.F.; MORAES, M.A.C.; SHIMOKOMAKI, M. Efeito da castração e salga na qualidade da carne de caprinos. Higiene Alimentar, v. 6, n. 22, p. 23-26, 1992.

[11] GARCIA, A.F.; MIZUBUTI, I.Y.; KANASHIRO, M.Y.; SHIMOKOMAKI, M. Intermediate moisture meat product: biological evaluation of charqui meat protein quality. Food Chemistry, n. 75, p. 405-409, 2001.

[12] GREENE, B.E.; CUMUZE, T.H. Relationship between TBA numbers and unexperienced panelists assessments of oxidized flavor in cooked beef. Journal of Food Science, v. 47, p. 52-58, 1982.

[13] LIRA, G.M.; SHIMOKOMAKI, M.; MANCINI-FILHO, J.; TORRES, E.A.F.S. Avaliação da oxidação lipídica em carne-de-sol. Higiene Alimentar, v. 14 , n. 68, p. 6669, 2000.

[14] MilTENBURG, G.A.J.; WENSING, Th.; SMULDERS, F.J.M.; BREUKINK, H.J. Relationship between blood hemoglobin, plasma and tissue iron, muscle heme pigment, and carcass color of veal. Journal of Animal Science, v. 70, n. 9, p. 2766-2772, 1992.

[15] MONTEIRO, E.M. Influência do cruzamento Ile de France x Corriedale (F1) nos parâmetros de qualidade da carne de cordeiro. São Paulo, 1998. 99p. Tese (Doutorado em Ciência dos Alimentos) - Faculdade de Ciências Farmacêuticas, Universidade de São Paulo (USP).

[16] NOGUEIRA, C.M.; ZAPATA, J.F.F.; SEABRA, L.M.J.; BARROS, N.N.; BORGES, A.S. Composição e propriedades físicas da carne ovina das raças Somalis Brasileira, Santa Inês e Morada Nova. In: CONGRESSO BRASILEIRO DE CIÊNCIA E TECNOLOGIA DE CARNES, 1., 2001, São Pedro. Anais...São Pedro, 2001. p. 191-192.

[17] PARDI, M.C.; SANTOS, I.F.; SOUZA, E.R.; PARDI, H.S. Ciência, higiene e tecnologia da carne. Goiânia:UFG, 2001. 623p.

[18] PIKUL, J.; LESZCZYNSKI, D.E.; KUMMEROW, F.A. Evaluation of three modified TBA methods for measuring lipid oxidation in chicken meat. Journal of Agricultural of Food Chemistry, v. 37, p. 13091313, 1989.

[19] ROÇA, R.O.Cura de carnes. http://www.fca.unesp.br/ tecnologiadecarnes.htm. 2002.

[20] SABADINI, E.; HUBINGERI, M.D.; SOBRAL, P.J.A.; CARVALHO JÚNIOR, B.C. Alterações da atividade de água e da cor da carne no processo de elaboração da carne salgada desidratada. Ciênc. Tecnol. Aliment., v. 21, n. $1,2001$.

[21] SAÑUdo, C.; SANCHEZ, A.; ALFOnSO, M. Small ruminant production systems and factors affecting lamb meat quality. Meat Science, v. 49, n. 1, p. 29-64, 1998.

[22] SILVA, D.J. Análise de alimentos: Métodos químicos e biológicos. 2.ed. Viçosa: Imprensa Universitária, 1990. 165p.

[23] SILVA, J. A. Tópicos da tecnologia de alimentos. São Paulo:Varela, 2000. 227p.

[24] SILVA SOBRINHO, A.G. Body composition and characteristics of carcass from lambs of different genotypes and ages at slaughter, Palmerston North, 1999. 54p. Report (PostDoctorate in Sheep Meat Production) - Massey University.

[25] SOUZA, X.R.; PEREZ, J.R.O.; BRESSAN, M.C.; BONAGURIO, S.; VIEIRA, J.O.; LEMOS, A.L.S.C. Características físico-químicas da carne de cordeiros do cruzamento Santa Inês e Bergamácia de diferentes sexos e pesos ao abate. In: SIMPÓSIO LATINO AMERICANO DE CIÊNCIA DE ALIMENTOS, 4., 2001, Campinas. Anais...Campinas, 2001. p.159.

[26 ] TRINDADE, M.A. Utilização da pré-cura de carne mecanicamente separada de frango (CMS) e suas vantagens. In: SEMINÁRIO AVANÇOS NA QUALIDADE DA CARNE E SEUS IMPACTOS NA INDÚSTRIA BRASILEIRA, 1., 2003, Piracicaba. Anais...Piracicaba, 2003.

[27] ZEOLA, N.M.B.L. Influência da alimentação nas características quantitativas da carcaça e qualitativas da carne de cordeiros Morada Nova. Jaboticabal, 2002. 65p. Dissertação (Mestrado em Zootecnia) - Faculdade de Ciências Agrárias e Veterinárias, Universidade Estadual Paulista (UNESP). 